\title{
De Max Scheler a Hans Reiner: OBSERVACIONES SOBRE LA TEORÍA DE LOS VALORES MORALES \\ EN EL MOVIMIENTO FENOMENOLÓGICO*
}

\author{
From MaX Scheler to Hans Reiner: \\ OBSERVATIONS ON THE THEORY OF MORAL VALUES \\ IN THE PHENOMENOLOGICAL MOVEMENT
}

Jean Héring

Universidad de Estrasburgo

Traducción**

Jimmy Hernández Marcelo

Universidad de Salamanca/Universidad de Turín

jim.her.mar@gmail.com

Las investigaciones sobre el problema de los valores morales en los fenomenólogos, sobre todo en Alemania, han estado dominadas durante mucho tiempo por la autoridad de la obra fundamental de Max Scheler: Der Formalismus in der Ethik und die materiale Werethik, aparecida hace casi medio siglo.

* Este artículo fue publicado originalmente como Jean Héring (1960). De Max Scheler à Hans Reiner. Remarques sur la théorie des valeurs morales dans le mouvement phénoménologique. Revue d'histoire et de philosophie religieuses, 40, 152-164. Agradecemos a Matthieu Arnold, directeur Revue d'histoire et de philosophie religieuses, por la autorización para la publicación de esta traducción.

** La presente traducción toma como fuente original francesa citada en la nota anterior. Las notas del traductor se introducen con un *. Las notas del autor - Jean Héring- siguen el orden de numeración del texto original en numeración arábica (1, 2, 3, etc.). Un agradecimiento especial a Paloma Vicente por las correcciones y sugerencias de traducción.

${ }^{1}$ Max Scheler, "Der Formalismus in der Ethik und die materiale Werethik". Jahrbuch für Philosophie und phañomenologische Forschung, I/2 (1913), pp. 405-565 y II (1916), pp. 21-428. 
Este standard work es tan conocido $-\mathrm{y}$, de hecho, está traducido en francés- ${ }^{*}$, que podemos contentarnos con recordar los dos puntos más importantes de su desarrollo. La obra se compone de dos partes; la primera -negativaconduce al ataque contra el formalismo de la moral de Kant, mientras que la segunda - positiva - se esfuerza en establecer una moral concreta ${ }^{2}$ sobre la noción de valor.

A continuación, añadimos, en honor del maestro, que no nos parece que haya confundido las nociones de valor y de $\operatorname{bien}^{2 b}$ (ni en moral ni en estética), como hacen los que asignan a los hombres el poder de "crear valores". Son los valores los que nos permiten distinguir los bienes de los males, las bellezas de las fealdades; únicamente las realidades en las cuales se manifiestan o se realizan los valores pueden, en ciertos casos, ser creados por nosotros ${ }^{3}$.

Pero sabemos también que Max Scheler se esforzó por establecer una jerarquía entre los valores en general (Jahrbuch, I/2, 507 ss.). En el nivel inferior se colocan los valores que podemos llamar hedonistas en sentido amplio (Die Werte des Angenehmen und Unangenehmen), por encima de éstos están, en primer lugar, los valores vitales (dentro de los cuales se encuentran también los valores de lo que es "noble" y "vulgar" —Edel und Unedel), le siguen los "valores del espíritu" (geistige Werte) entre ellos encontramos la belleza, la justicia, la verdad o -dicho con más exactitud- el conocimiento de la verdad. Finalmente, en la cima, encontramos los valores de santidad y de su opuesto (Werte des Heiligen und Unheiligen).

Lo que hay de curioso en este cuadro es la ausencia de los valores morales propiamente dichos, ya que nadie pensaría que su riqueza quedaría agotada por la noción de justicia. Asimismo, el acto moral (o inmoral) no puede consistir en optar por (o contra) un valor moral. Se trata simplemente de elegir entre un valor superior y otro inferior. La acción buena es la que opta por la superior y la

\footnotetext{
* Jean Héring cita la traducción francesa de 1955. A continuación la referencia completa: Max Scheler Le formalisme en éthique et l'éthique matériale de valeurs: Essai nouveau founder un personnalisme éthique, Paris: Galimmard, (1955).

2 Es mejor no traducir "material" (alemán) por "material".

${ }^{2 b}$ Según parece pensar Hans Reiner en "Über Grundbegriffe Und Methode Der Ethik". Zeitschrift für Philosophische Forschung, IX/3 (1955), 492, nota 9. Véase Max Scheler, "Der Formalismus in der Ethik und die materiale Werethik", primera parte, $\S 2$.

${ }_{3}$ Pensamos que para una sana teología los valores tienen su fuente en Dios. Por ello, una de las primeras "reformas" introducidas por las bestias apocalípticas que quieren ponerse en lugar de Dios, es la fijación arbitraria de un código moral y estético.
} 
mala la que opta por la inferior y el valor superior se supone que es reconocible por ciertas particularidades (Jahrbuch, I/2, 492 ss.).

Antes que nada, señalamos - junto a la mayoría de críticas- que el acto moral no siempre presupone una elección entre dos valores. En muchos casos, se trataría simplemente de aceptar o de rechazar un único valor. De este modo, yo puedo decidir - sí o no- ayudar a un indigente sin ninguna competición entre dos valores. Otras objeciones se presentarán a continuación.

El primer paso decisivo más allá de Max Scheler fue hecho, salvo algún error, por Dietrich von Hildebrand con dos contribuciones importantes en el Jahrbuch de Husserl ${ }^{4}$. Estas disertaciones contienen muchos análisis valiosos, en modo alguno obsoletos, y sí más fecundos que tantos razonamientos abstractos sobre "el valor, el ser y la conciencia". Ante todo, Dietrich von Hildebrand ha restablecido el puesto de los valores específicamente morales que no se encuentran en el cuadro axiológico de Max Scheler. Después, asumiendo la crítica al formalismo de Kant, añade una crítica expresa a su voluntarismo. En efecto, no hay ninguna razón para hablar de la voluntad como la única "portadora" posible de los valores morales, como ha hecho el filósofo de Königsberg en su elogio de la voluntad buena ${ }^{5}$. La bondad, la nobleza, la pureza de corazón son virtudes que encarnan valores en sí mismos, independientemente de cualquier relación con la voluntad. Si se proclama "bienaventurados los puros de corazón", no se pregunta si es por un esfuerzo de voluntad o por algún otro medio que explique la posesión de esta cualidad. Y si se quisiera plantear esta pregunta, el Evangelio respondería sin duda que se trata de un don del cielo (ilo cual no disminuiría en nada su valor!). Aquí se puede incluso preguntarse si la relación exclusiva entre el esfuerzo y el ethos en Kant ( $y$ en muchos otros) no ajusta, en última instancia, a una herencia de un cierto fariseísmo que únicamente valora el esfuerzo meritorio del hombre. En todo caso, estaremos de acuerdo con Dietrich von Hildebrand que enseña -

\footnotetext{
${ }^{4}$ Dietrich von Hildebrand, "Die Idee der sittlichen Handlung". Jahrbuch für Philosophie und phänomenologische Forschung, III (1916), pp. 126-252 y "Sittlichkeit und ethische Werturteile". Jahrbuch für Philosophie und phañomenologische Forschung, V (1922), pp. 462-602. Sabemos que este autor es un discípulo de Husserl y de Scheler de la época anterior a 1914.

${ }_{5}$ Por razones evidentes, no se debe traducir guter Wille por "buena voluntad".
} 
al principio de su primera disertación-, citando a Lutero, que es el valor de la persona lo que condiciona el acto moral y no a la inversa.

Otro progreso de estas contribuciones consiste en haber señalado la importancia - para la moral- de ciertos valores que no están relacionados ni con acto ni con la actitud, ni con la persona, sino con situaciones (Sachverhalte). Hay "estados de cosas" justas o injustas, regocijantes o desoladoras, sanas o insanas. Desafortunadamente, el autor no realiza análisis ni cuadros de estos Sachverhaltswerte. Se limita a constatar que, dentro de estos valores, los hay que son moralmente importantes (sittlich relevant) - cuya realización sería el objetivo del acto moral- para el estudio del tema al que está destinada disertación.

Pero también se trata de distinguir, mejor de lo que Max Scheler había hecho, los diferentes modos de percibir un valor; éstos pueden ser puramente afectivos (das Wert-Fühlen) -el único modo del que habla Max Scheler- o al contrario, sostenidos por un conocimiento claro. Sólo en el segundo caso podemos verdaderamente dar cuenta de nuestra experiencia. Asimismo, se debe distinguir entre las diferentes reacciones (Max Scheler: Antwort-reaktion), más bien afectivas o voluntarias, del valor positivo o negativo (real, deseado o temido) que se ha percibido.

Estos análisis son llevados más lejos en la segunda disertación. Es difícil resumir estas investigaciones fenomenológicas cuyo interés reside, de hecho, tanto más en el método concienzudo que en los resultados, los cuales no son necesariamente espectaculares como los de los edificios gloriosos, pero bastante frágiles, de la Weltanschauungsphilosophie (término, por suerte, intraducible).

Nos gustaría aquí atraer la atención sobre dos temas que Dietrich von Hildebrand ha desarrollado de una manera particularmente profunda y original. En primer lugar, el fenómeno de la ceguera axiológica. Hay sujetos insensibles a los valores morales, así como hay otros que carecen de órganos para la percepción de los valores estéticos ${ }^{6}$.

\footnotetext{
${ }^{6}$ El autor piensa, en efecto, que, al contrario de la ceguera estética, la ceguera moral es siempre, en cierta medida, imputable a la persona, la cual no habría tenido que "dejar llegar las cosas hasta allí".
} 
Llevado al extremo, la ceguera se extiende a todos los valores morales. Casos similares no sólo se encontrarían en personajes groseramente egoístas. Conocemos el ejemplo de sujetos bien cultivados, en quienes el sentido estético puede estar bien afinado, pero que no se dan cuenta de sus faltas de cortesía frente a su propia familia. Otras personas ven y comprenden los valores circunscritos por los términos "justicia" u "honestidad", pero no saben apreciar la belleza de un acto de generosidad o de perdón?.

Otro fenómeno, bastante extendido, es presentado por lo que el autor llama Subsumptionsblindheit, es decir, la incapacidad de constatar, in concreto, la presencia de un valor, pero que por principio se reconoce. iCuántos sujetos pueden inflamarse por la "filantropía" sin conmoverse por la miseria de sus vecinos!8

No hace falta decir que la ceguera es muy diferente al desprecio consciente de un valor moral positivo, ya sea por principio o in concreto. Es únicamente aquí donde se realizan las actitudes y los actos que ameritan el calificativo de específicamente inmorales.

Otros desarrollos, sobre los que no podemos insistir aquí, estudian las diferentes variedades de profundidad de las reacciones respecto de los valores.

Mucho después, el autor -emigrado a los Estados Unidos- publica una importante obra en inglés titulada Christian Ethics ${ }^{9 *}$, en la que retoma estas cuestiones. Este título no debe inducirnos a error. Dietrich von Hildebrand no intenta en absoluto oponer una "moral cristiana" a una "moral profana". Sino que cree que el cristianismo puede ayudarnos a ver o a ver mejor los valores morales, 0 a darles una significación nueva. No obstante, la percepción intuitiva es siempre la base de la moral.

\footnotetext{
${ }^{7}$ A veces, la ceguera es causada por hábitos de pecar sin arrepentimiento sincero. En cuanto a los casos en los que el arrepentimiento parece imposible, ya que la persona ha cometido crímenes con una completa buena conciencia (por obedecer las órdenes de un jefe), el autor no parece haber podido imaginarlos.

${ }^{8}$ Casos paradigmáticos han sido ofrecidos por Charles Dickens, quien sabe representar a mujeres buenas que trabajan de modo fanático por la educación de los pequeños negrès en África, sin ser afectadas por la manera lamentable en la que son criados sus propios hijos.

9 Publicado en Londres en 1952 con el Imprimatur del cardenal Spellmann.

* El libro se publicó en Estados Unidos de América en 1953. El Cardenal Francis Spellman era arzobispo de Nueva York. La referencia completa del libro es: Dietrich von Hildebrand (1953), Christian Ethics, New York, David Mckay Company (Trad. Esp. de Salvador Gómez Nogales: Dietrich von Hildebrand (1962), Ética cristiana, Barcelona: Herder).
} 
Al analizar la esencia de los valores morales de una manera más profunda que en sus publicaciones anteriores, estudia ahora especialmente su carácter vinculante e independiente respecto de nuestro buen querer. Sin embargo, no lleva aún muy lejos el análisis de los "valores de situaciones" (Sachverhaltswerte). Nos limitamos a decir que, en este campo, hay valores que tienen una incidencia moral (moralische relevante Werte) y señalamos el progreso real realizado por la distinción clara entre valores ontológicos y valores morales (p. 129 y siguiente). Si se habla de la superioridad del hombre en relación al animal o del animal respecto a la planta, ésta no tiene nada que ver con un nivel moral. Para el hombre, se pensaría sin duda en cierto grado de inteligencia, de libertad, de sensibilidad religiosa ${ }^{10}$. Pero no hace falta decir que desde el punto de vista moral, los animales pueden dar prueba de cualidades morales, tales como la fidelidad o la valentía, los cuales podrían servir de modelo para muchos humanos. A lo sumo se puede estimar que ciertas cualidades humanas, como la libertad, nos imponen la obligación de llevar a cabo un estándar moral superior, sin que éstas sean virtudes.

Esta distinción entre el orden ontológico y el orden moral debería poner fin a algunas confusiones lamentables, las cuales con frecuencia han contaminado la moral, tanto en Oriente como en Occidente. Lo que separa indudablemente al hombre de Dios, no es su distancia ontológica, su situación "limitada en el tiempo y en el espacio" (sabemos de lo que se habla), sino su pecado. Y éste no se cura con ejercicios místicos o el desarrollo de facultades psíquicas superiores.

En lo que concierne a los valores morales, el autor insiste en la distinción, entrevista ya por Scheler, entre valores en sí y valores para mí (p. 34 y siguiente). Él no piensa, por tanto, que se deba reservar el término valor al primer grupo, sino que éste comprende valores más objetivos y más constreñidos. No obstante, como lo desarrollará Hans Reiner, la cuestión no es tan simple, pues hay un tercer grupo: los valores para los otros. Pero es cierto que al elegir entre las dos clases de valores distinguidas por Dietrich von Hildebrand, sólo la primera produce en nosotros un sentimiento de responsabilidad y cuyo desprecio crea en nosotros el fenómeno de la mala conciencia. Naturalmente, esto no quiere decir que todo valor moral pueda ser realizado por nosotros.

\footnotetext{
${ }^{10}$ Pero no en la posición vertical de la que tanto se presume y que comparte con los avestruces y los canguros y con la mayoría de plantas.
} 
Añadimos igualmente - esto no lo dice el autor- que no sólo son los individuos, sino también las colectividades las que pueden y deben sentirse responsables de ciertas situaciones de índice moral positivo o negativo, lo cual implica nuevamente en cierto modo la responsabilidad de los individuos en tanto miembros de una colectividad ${ }^{11}$.

No hace falta decir que los valores circunscritos por el término virtud plantean aún otros problemas. Éstos valores son "objetivos" en el sentido de que son valores en sí, pero de los que no somos necesariamente responsables, según lo hemos ya indicado. No obstante, podemos sentirnos responsables de nuestros vicios, así como de nuestra decadencia que los engendra. Sea como fuere, es difícil tener una conciencia clara de nuestra caída.

El autor no se pronuncia explícitamente sobre la famosa teoría de la infalibilidad de la conciencia, pero al parecer no cree mucho en ella, puesto que enseña la perfectibilidad de la conciencia y su mutismo en los casos de ceguera axiológica. Además, en sentido estricto, la conciencia no nos habla de los malas acciones de los demás.

Para concluir, creemos que hemos dado una idea de los progresos realizados en el estudio de los valores morales a través de los análisis fecundos de Dietrich von Hildebrand. Sin embargo, numerosos problemas quedan por estudiar o volver a estudiar. Pensamos que son especialmente los estudios de Hans Reiner los que merecen nuestra atención ${ }^{12}$.

Las publicaciones de Hans Reiner que en este tema entran en consideración son: Das Prinzip von Gut und Böse ${ }^{13}$ y Pflicht und Neigung ${ }^{14}$.

Como Scheler, Reiner emprende (especialmente en la segunda obra indicada) una fuerte crítica, pero mejor documentada de la posición de Kant, dando cuenta también de un modo más detallado de la polémica de Schiller de la que no podemos hablar aquí.

\footnotetext{
${ }^{11}$ No se debería distinguir "moral individual" y "moral social" oponiendo el hombre solitario (que no existe) y el hombre que vive en sociedad. La moral social debe fijar los derechos y valores de las colectividades, que son distintos a los de los individuos.

12 Hans Reiner, discípulo de Husserl de la segunda generación. Actualmente [en la fecha del artículo] es, junto a Eugen Fink, profesor de filosofía en Friburgo.

${ }^{13}$ Hans Reiner (1949). Das Prinzip von Gut und Böse, Freiburg: Karl Alber.

${ }^{14}$ Hans Reiner (1951). Pflicht und Neigung. Die Grundlagen der Sittlichkeit, Meisenheim: Hain.
} 
Pero, por otro lado, también rechaza - como Hildebrand- la famosa interpretación scheleriana del acto moral como una elección entre un valor superior y uno inferior (Prinzip, p. 3 y siguiente). Según hemos ya indicado, en muchos casos no se trata de elegir, sino de decir sí o no a un único valor. En otros, se trata de elegir entre dos o más valores de la misma esfera. Ante uno y otro de estos grupos de situaciones, la moral de Scheler está completamente desarmada. Es difícil clasificar en su cuadro el valor de una vida humana en otro lugar que en grado de los valores biológicos, inferiores a los valores artísticos, como hemos visto. Sin embargo, dudamos en calificar de inmoral la acción de un bombero que ha preferido salvar a un niño (tal vez insoportable) antes que la pintura de un artista (tal vez muy valiosa). Ya que aquí, tal como lo ha señalado Hans Reiner ${ }^{15}$, no es el nivel del valor el que cuenta (incluso si se supone exacta la jerarquía de Scheler), sino su fuerza particular que él denomina Dringlichkeit (literalmente: "urgencia").

En la práctica, la elección es con frecuencia espinosa. Hans Reiner cita los casos de un arquitecto encargado de la reconstrucción de una ciudad destruida por la guerra, quien se pregunta si tiene el derecho de volver a edificar el teatro ("bien artístico-técnico", según el esquema de Scheler) antes de haber procurado a todos los ciudadanos una habitación decente. Si opta por el teatro, Scheler tendrá que alabarlo de todos modos. En cuanto a nosotros, tal vez lo culpemos, pero ¿tendríamos el derecho de denominar su elección como virtuosa o malvada (según nuestro enfoque)? Evidentemente, no. Por ello Hans Reiner introduce aquí una distinción capital ${ }^{16}$ : distingue la oposición entre actos buenos y malvados -Gut und Böse- de la oposición entre actos justos y equivocados - Richtig und Falsch (Prinzip, p. 5). En efecto, parece injusto acusar de maldad al arquitecto que habría elegido mal.

Pero si el arquitecto cediese a los amantes del teatro por razones egoístas, entonces - piensa Reiner- su elección sería "malvada" (Böse). Preferiríamos llamarla "mala" (Schlecht), por razones que explicaremos más adelante.

${ }^{15}$ Nicolai Hartmann, suficientemente influenciado por el movimiento fenomenológico, había presentado observaciones análogas. Pero un estudio de este pensador nos conduciría bastante lejos de aquí. En concreto se debería volver a leer su Ethik (Berlin, 1949).

${ }^{16}$ Algunos críticos se mofan de los fenomenólogos por su "manía" de las distinguo. Pero ¿cómo salir de la confusión y de las simplificaciones sin desentrañar las esencias? 
Sin embargo, se podría presentar una objeción: se entiende, se podría decir, que la preocupación por el beneficio no es suscitado por el respeto de un valor en sí, sino sólo por un valor para el sujeto; por tanto, sin valor moral. Pero ¿la comodidad de los habitantes no presenta también un valor subjetivo? ¿Se debería hablar de bienes justificados y de otros que no lo son? El autor piensa que se debe introducir aquí una separación más radical: entre los valores para mí (Eigenwerte) y los valores para los otros (Fremdwerte) ${ }^{17}$ (Prinzip, p. 9 y siguiente).

Estamos aquí lejos de la famosa "ley general" del kantismo. Entonces, no son sólo los valores absolutos, sino también los valores relativos a los otros, los que merecen ser llamados "objetivos", es decir, "arrojados frente a nosotros". Hans Reiner, por tanto, llega a los dos cuadros siguientes:

\section{PRIMER CUADRO:}

I. Valores relativos:

a) Valores relativos a mí

b) Valores relativos a los otros

II. Valores absolutos (en sí)

\section{SEGUNDO CUADRO}

I. Valores subjetivos (=relativos a mí):

II. Valores objetivos:

a) Valores relativos a los otros

b) Valores absolutos

(Prinzip, ibíd., y Pflicht, p. 141)

¿Qué es lo que caracteriza, en definitiva, a los valores absolutos? La imposibilidad de dejarlos de lado. No sucede así con el otro grupo de valores objetivos, a saber, los valores relativos al prójimo. Éste puede renunciar al bien que tendría un valor para él, por ejemplo, la ayuda que nosotros quisiéramos brindarle. Entonces, la llamada del valor enmudece. Al contrario, las exigencias que emanan

17 Este muro de separación ha pasado desapercibido, salvo error, a Hildebrand y por N. Hartmann, sólo Scheler hizo una muy breve alusión. Cf. Max Scheler, "Der Formalismus in der Ethik und die materiale Werethik". Jahrbuch für Philosophie und phanhomenologische Forschung, I/2 (1913), p. 503. 
de los valores absolutos son irrefutables (Die von den absoluten Werten ausgehnden Forderungen sind unaufhebbar) ${ }^{18}$.

Sin duda, algunos espíritus curiosos reclamarían un criterio más simple que permita distinguir, de una vez por todas, los valores absolutos de los otros, como se distingue dos razas humanas o animales. El fenomenólogo responderá que a menos que se ponga a la ética en el remolque del utilitarismo o de una construcción filosófica más o menos arbitraria, no hay otro principio de conocimiento para los valores fundamentales que la intuición "del corazón" (que puede perfectamente ser a priori).

Esto nos conduce a la cuestión de la conciencia moral personal (Gewissen) (véase especialmente el capítulo IV de Pflicht). Ésta aparece como el lugar geométrico de la manifestación de una exigencia moral que nos concierne personalmente. Hace falta señalar que el terreno de este género de conciencia es más estrecho que el de la conciencia moral tout court, la cual también juzga el comportamiento de los otros $^{19} \mathrm{y}$ de cierta manera las situaciones que escapan a nuestro poder.

Por otro lado, el análisis fenomenológico confirma la idea popular de que la conciencia moral, en sentido fuerte, emana de una capa de nuestro yo distinta de la que es la sede de nuestra libertad y de nuestra voluntad. El autor identifica ésta con la vida del espíritu y aquélla con la vida del alma, la cual contiene, de algún modo, nuestra materia psíquica - acercándose un poco a la antropología de Alexander Pfänder 20 .

¿Qué piensa Hans Reiner de la tesis a menudo sostenida sobre el carácter puramente negativo de la conciencia, la cual sólo sabría condenar el mal (a veces

\footnotetext{
${ }^{18}$ Hans Reiner (1949). Das Prinzip von Gut und Böse, Freiburg: Karl Alber, p. 13.

19 Nuestro maestro Jean Monnier resalta a veces en sus sermones la persistencia de la facultad de juzgar válidamente a los otros, incluso en caso de atrofia de nuestra conciencia personal.

${ }^{20}$ Véase especialmente Alexander Pfänder (1933), Die Seele des Menschen, Halle, Max Niemeyer. Su libro sobre el sentido de la vida es bastante instructivo para los moralistas: Alexander Pfänder (1948), Philosophie der Lebensziele, Goittingen, Vandenhoeck \& Ruprecht. Pero supone resueltos los problemas elementales sobre los que aquí hablamos. En último momento, se nos anuncia una nueva publicación fenomenológica tricotomista: Cornelius Anthonie Van Peursen (1959), Leib, Seele, Geist. Einfuhrung in eine phähomenologische Anthropologie, Guitersloh: G. Mohn.
} 
sólo después del hecho) sin empujarnos hacia el bien? Daremos la razón a nuestro autor, que rechaza esta manera demasiado simplista de ver las cosas. La voz de la conciencia puede muy bien empujarnos hacia una decisión moral exigida por la apelación a un valor, sin pasar necesariamente por el desvío de un rechazo previo de la mala acción o por la indignación contra un mal que debe ser eliminado (véase Pflicht, p. 116). Una madre podría complacer a su hijo sin darse cuenta de que la omisión de esta acción sería (quizás) mala o de que el niño estaba sumergido (quizás) en una tristeza por curar.

En cuanto al problema de la infalibilidad de la conciencia, al que Dietrich von Hildebrand había aportado una contribución indirecta mediante sus análisis sobre la ceguera moral, Hans Reiner tampoco habla de él de manera explícita. Pero, al leer entre líneas, hemos tenido la impresión de que él no negaría la posición de la Carta a los hebreos en la que se recomienda ejercitar nuestros órganos (es decir, nuestras facultades intuitivas), destinados a distinguir el bien y el mal ${ }^{21}$.

Los desarrollos que acabamos de resumir serían ampliamente suficientes para cimentar la moral sobre una base distinta del formalismo kantiano. Pero podría subsistir un malentendido: la insistencia con la que el autor resalta la apelación inexorable de los valores morales absolutos podría hacer creer que da razón al filósofo de Königsberg sobre otro punto, a saber, la lucha inevitable entre el "deber" y las "inclinaciones" (Pflicht und Neigung). Ahora bien, esto no es así. Una de las partes más interesantes de su libro principal está incluso consagrado a esta cuestión, como el título lo hace prever (véase especialmente §§ 25-32). En primer lugar, el autor protesta contra la identificación, algo precipitada, de las actitudes (Verhalten) egoístas con aquellas al servicio de una inclinación (Neigung). El seguimiento de un fin egoísta puede incluso conducirnos (como algunos objetivos morales) a violentar nuestras inclinaciones. De este modo, un sujeto de temperamento indolente debería combatir su pereza para ganar dinero.

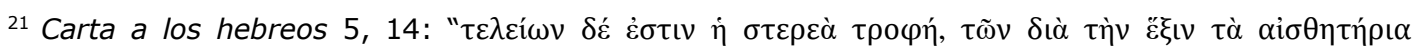

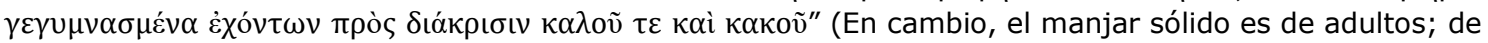
aquellos que, por la costumbre, tienen las facultades ejercitadas en el discernimiento del bien y del mal). Este versículo cuya importancia no siempre ha sido percibida, conduce de algún modo a la cumbre de la moral anti-heteronomista del Nuevo Testamento, pero sin dar razón a J- J. Rousseau. 
Añadiremos que ni Kant, ni la mayoría de sus sucesores (salvo los psiquiatras) parecen haber considerado suficientemente la ambivalencia afectiva de ciertos actos por los cuales experimentamos simultáneamente ánimo y repugnancia. Incluso si una de las dos inclinaciones opuestas vence, la otra no está necesariamente aniquilada. La literatura está llena de ejemplos de mujeres que experimentan a la vez amor y repulsión por ciertos individuos, la primera afección que puede subsistir aun cuando la otra ha ocupado el lugar superior ${ }^{22}$.

Lo que es todavía más falso es creer con Kant que "deber" e "inclinación" se excluyen necesariamente. Cuando me gusta el bien que quiero realizar (wenn ich es gern tue), mi buena acción no se desvaloriza. Incluso existe, piensa Hans Reiner con Schiller, un amor por el deber como tal, independientemente de la situación particular (Neigung zu der Pflicht), y quien sabe si Schiller tuvo algo de razón al considerar esta motivación como un ideal ${ }^{23}$. Sin embargo, no se puede dudar de que el amor por el deber (lo que Hans Reiner, salvo error, no ha indicado) parece, a pesar de todo, revelar una coloración distinta a cualquier otra inclinación. Sobre este punto Kant ha visto un fenómeno particular al otorgar un lugar especial -lejos de todas las "afecciones" - a lo que denomina Achtung fürs Gesetz (respeto por la ley), hechas naturalmente todas la reservas sobre la concepción legalista de su moral.

De igual forma, daremos la razón pericialmente a Kant en el sentido de que, rebus sic stantibus, los conflictos entre el "deber" y las "inclinaciones" son frecuentes, aunque no necesarios.

22 A la inversa, hay hombres que sin estar realmente locos, ven en las mujeres algo como un mysterium tremendum atque fascinosum (como si ellas fuera divinidades). El "por" y el "contra" a veces se fusionan de manera curiosa.

${ }^{23}$ Cf. Friedrich Schiller (1793), Uber Anmut und Würde, Leipzig. Para el uso de los lectores que los hayan olvidado, nos permitimos recordar dos dísticos satíricos de Schiller sobre la moral de Kant.

Pregunta: "Gerne dien' ich den Freunden, doch tu' ich es leider mit Neigung, und darum wurmt es mir (sic!) oft, dass ich nicht tugendhaft bin".

Respuesta: "Da bleibt kein anderer Rat, du musst suchen sie zu verachten und mit Abscheu alsdann tun, was die Pflicht dir gebeut".

Lo cual se podría traducir algo así como:

"Me gusta muchos servir a mis amigos - desafortunadamente- lo hago con placer y por ello a menudo tengo remordimientos, pues me digo a mí mismo que no soy virtuoso".

"Entonces, sólo te queda una cosa por hacer: intenta despreciarlos para luego realizar con disgusto lo que el deber ordena". Cf. Friedrich Schiller, Die Philosophen (en Poesías del tercer período). 
Sin embargo, no olvidemos - como lo señala Hans Reiner- que a veces nuestro interés (preocupado por los valores subjetivos) va a la par con la exigencia moral. Un propietario, que habita una planta de su casa y quien hará oportunamente las reparaciones necesarias al techo, servirá a los intereses de sus inquilinos y los suyos propios ${ }^{24}$. El valor moral de su decisión perdería su valor sólo si la preocupación de su propio interés hubiera sido el principal o el único móvil del acto. En este caso, el bien hecho a los otros no le sería imputado.

Del mismo modo, se debe preguntar si se es responsable o no de las malas consecuencias no previstas, pero previsibles, de un mal acto (véase p. 178 y siguiente). Parece que sí: si hago descarrilar un tren para matar a un adversario político, yo sería responsable también de la muerte de los demás pasajeros accidentados, incluso si no lo había pensado; pues esta consecuencia era previsible ${ }^{25}$. Por tanto, no hay equivalencia moral entre los efectos buenos y malos de una acción moralmente negativa.

Tercer caso: una acción buena ha entrañado buenos efectos suplementarios no previstos. Si rescato a un bebedor de la embriaguez y que luego de este milagro, su mujer renuncia a suicidarse, yo le habría de hecho salvado la vida. Pero ésta feliz consecuencia no prevista no se me tomaría en cuenta. Por tanto, aquí se da una curiosa equivalencia de los efectos buenos de una acción mala o buena. Todas estas verdades se muestran, de hecho, en la intuición directa y no de cualquier intuición.

Pero ¿qué pensar de los casos en los que una acción legítima entraña o implica una falta de respeto por los valores positivos? Si un ayuntamiento, para evitar el gran desastre de una inundación, hace evacuar precipitadamente una localidad y con medio improvisados ¿no sería éste responsable de la muerte de

${ }^{24}$ La mayoría de ejemplos aquí citados han sido elegidos por el autor de este estudio crítico.

${ }^{25}$ La situación es análoga en el caso de omisión de una acción moralmente exigida. ¿Si nos rehusamos a salvar a una persona presa de un incendio no seríamos también responsables de la muerte del equipo de salvamento, que habiendo llegado después de nosotros, habría llegado demasiado tarde? Se debe anotar aquí que la cuestión desborda la esfera de las acciones derivadas de la moral. Fanáticos que escalan la cara norte del Eiger, sin guía y sin preparación suficiente, indudablemente sólo cometen una imprudencia y no una maldad. No obstante, su conducta deberá ser considerada inmoral indirectamente (par ricochet), ya que su fracaso, que pondría en peligro la vida de sus rescatadores, era previsible. 
personas mayores o discapacitados que no soportarían el viaje en estas condiciones? ${ }^{26}$ Hans Reiner piensa que en los caso de este género (los que, en efecto, muestran la acción moralmente justa o equivocada, antes que una acción buena o mala), la colectividad debería aceptar esta responsabilidad. Pero rechaza enunciar una solución general. Imposible no examinar los pros y contras en cada caso particular. El autor espera de los demás el reproche de elaborar una "casuística"; pero la acepta valientemente (Pflicht, p. 174).

La "casuística" es inevitable para resolver el famoso problema del "conflicto de deberes" (Pflichten-Kollision), que en absoluto es un falso problema, como pensaba Schleiermacher. Ciertamente, este pensador tenía razón en pensar que siempre hay, en principio, un deber más urgente que otro. Pero el descubrimiento de este valor necesita de una elección que a menudo estaría precedida de una lucha. Quod erat demonstrandum ${ }^{27 *}$.

Dos observaciones más para terminar este estudio, a la vez demasiado extenso y demasiado breve, pero aceptamos este reproche debido a las dificultades inherentes a la tarea del que reseña.

Hemos ya indicado que queríamos reemplazar, en la mayoría de casos, el término "malvado" (méchant-böse) -empleado por Hans Reiner para todos los actos inmorales- por el de "malo" (mauvais-schlecht). En efecto, pensamos que el término de böse se utiliza de manera adecuada cuando el sujeto desprecia los valores positivos, no por amor a sí mismo, sino por amor a los valores negati$\operatorname{vos}^{28}$, por ejemplo, al entusiasmarse por la injusticia o el odio de los valores

${ }^{26}$ Visto desde el ángulo de la religión del Nuevo Testamento, esta constante imposibilidad de hacer el bien "sin ensuciarse las manos" (el "semper peccator" de Lutero toma aquí un sentido muy especial) es una prueba de la caída que no sólo ha afectado nuestro corazón y nuestro cuerpo, sino también ha estropeado la "relojería" del mundo que los discípulos de Leibniz consideran perfecta.

27 Por nuestra parte, añadiremos además que Scheler no se había equivocado al advertimos de las falsas situaciones de quienes se comprometen precipitadamente en "conservar ambos". Pero entonces, se trata de un conflicto del que somos responsables, lo cual no es el caso en las situaciones más normales.

* En la nota anterior Héring utiliza la expresión "Ménager la chèvre et le chou" que hace referencia al hecho de satisfacer a dos personas u opiniones opuestas. Hemos traducido por "conservar ambos". Este "ambos" (la chèvre et le chou) se refiere a los dos valores que entran en conflicto.

${ }^{28}$ De una manera general, nos parece que los moralistas no han estudiado suficientemente los valores negativos y su función en la inmoralidad individual o colectiva (tal vez exceptuados los teólogos, pero por lo general los han pensado únicamente bajo un ángulo determinado). En cuanto a las mentes recién llegadas persisten en no ver en la presencia de los valores negativos nada distinto que la ausencia de valores positivos. Nosotros pensamos que nadie se los toma en serio. 
positivos ("detestamos la objetividad", "no puedo soportar la palabra humani$\left.d a d^{\prime \prime}\right)$. Ningún interés egoísta interviene aquí de modo esencial. Al contrario, cuanto más desinteresado es el amor al mal, más aparecerá el fenómeno de la maldad en su pureza.

Esto no impide que algunos sujetos también amen los valores subjetivos negativos, por ejemplo, sus propios sufrimientos físicos. Sin mencionar los casos verdaderamente patológicos, se podría pensar en algunos que se flagelan o en la mujer que "le gusta ser golpeada". ¿Existe también una inclinación para el sufrimiento psíquico? Quizás sí, aunque estos casos parecen raros y complejos. De todos modos, sería difícil decidir si este amor por el propio sufrimiento debe ser calificado de inmoral29. Éste es, en todo caso, incomparablemente menor que la inclinación por infligir males a los otros -en conformidad a la no-equivalencia del yo y del tu que una moral esquemática muy a menudo ha olvidado.

A continuación, se permitirá expresar una decepción. Nos hubiera gustado que el autor estudiara más de cerca los valores absolutos cuyo portador (como dice Hildebrand) no es el acto, sino la cualidad del sujeto. ¿La definición de valores absolutos, como los que se nos dirigen en una llamada irrefutable, les es igualmente aplicable? En algunos casos, con seguridad sí. De este modo, el valor positivo de una virtud, antes de desarrollarse en un niño, se dirige - se podría decir - a una llamada al educador. La virtud de un héroe puede empujar a sus admiradores a esforzarse para ser como él. Pero no se debería, en todos los casos, colocar la virtud en el terreno de la libertad y de la responsabilidad. Como lo hemos ya indicado, una cualidad, por ejemplo la belleza de un alma, conservaría su valor, incluso si ésta resulta ser innata u otorgada por la gracia. Para ser honestos, hemos tenido la impresión de que el autor tiene la tendencia de restringir el dominio de la moral (Sittlichkeit) al del campo de la libertad ${ }^{30}$. Confesamos no comprender bien el porqué.

Hemos llegado al final de este viaje. No hemos ocultado nuestra aprobación a la mayoría de las tesis aquí resumidas (parcialmente) y discutidas. A menudo incluso hemos superado las enseñanzas de Dietrich von Hildebrand y sobre todo

${ }^{29}$ Y la ayuda que se les pide a los otros que brinden. Ahí se trata, en primer lugar, de saber si ésta es dada únicamente para "ofrecer un servicio", o para satisfacer los malos instintos. Pero con esto no todo está dicho.

${ }^{30}$ En Pflicht (p. 82), dice incluso lapidariamente: "la existencia moral está vinculada a la libertad" (nur wo Freiheit ist, ist Sittlichkeit). 
de Hans Reiner, pero esperando habernos mantenido en las líneas que se desprenden de éstos. Pero, incluso los lectores que adoptasen una actitud más reservada, quizás estarían de acuerdo en el profundo interés de estos análisis fenomenológicos, aunque no sean "trascendentales" a la manera de Husserl. 